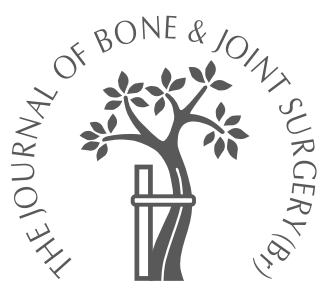

P. F. Rosenfeld, S. A. Budgen, T. S. Saxby

From the Brisbane Foot and Ankle Centre, Brisbane, Australia

\title{
Triple arthrodesis: is bone grafting necessary?
}

\author{
THE RESULTS IN 100 CONSECUTIVE CASES
}

Our aim was to evaluate the results of triple arthrodesis, performed without the use of supplementary bone graft. We carried out a retrospective review of 100 consecutive triple arthrodeses. All the operations had been performed by the senior author (TSS) using a standard technique. Only local bone graft from the excised joint surfaces had been used, thereby avoiding complications at the donor site.

The mean age of the patients at surgery was 58 years (18 to 84 ). The mean time to union was 5.1 months ( 3 to 17). There were 75 good, 20 fair and five poor results. There were four cases of nonunion.

Our study has shown that comparable rates of union are achieved without the need for supplementary bone graft from the iliac crest or other donor site.

A triple arthrodesis is a simultaneous fusion of the subtalar, talonavicular and calcaneocuboid joints. Nonunion is a common cause of a poor outcome. In 1965, Wilson et $\mathrm{al}^{1}$ stated that "the outstanding cause of failure was the formation of pseudarthroses". Historically, the incidence of nonunion has been significant ranging from $10 \%$ to $23 \% .^{1-3}$ In a review of 301 cases, Wilson et $\mathrm{al}^{1}$ suggested three factors which could predispose to nonunion, namely, the absence of internal fixation, poor bony apposition and early weight-bearing. However, with the introduction of bone grafting and modern methods of internal fixation, the rates of nonunion have been reduced to between $0 \%$ and $4 \%{ }^{4-8}$

The most common site for bone grafts is the anterior iliac crest, but the proximal tibia, greater trochanter, calcaneum and talus have also been used for triple fusions. ${ }^{4,9}$ However, the harvesting of iliac crest bone incurs considerable donor-site morbidity and there is a significant incidence of pain at the donor site. Minor complications occur in up to $10 \%$ of cases and major complications include deep infection, vascular injury, deep haematoma, nerve injury, abdominal hernia and fracture of the iliac wing. ${ }^{10,11}$

The number of patients receiving bone grafts is inconsistent, with no comment on the indication. Our aim therefore was to evaluate the results of triple arthrodesis without the use of supplementary bone graft.

\section{Patients and Methods}

Between January 1993 and July 2001, the senior author (TSS) performed 112 triple arthrodeses on 108 patients. We carried out a retrospective review of these patients. Exclusion criteria were revision procedures (seven), and those who had bone grafting from the iliac crest or another donor site (five). The indications for bone grafting were failure of bony apposition at the fusion site (four) with more than a $2 \mathrm{~mm}$ gap at the time of surgery, or the use of a structural bone graft to correct deformity (one). The remaining 96 patients (100 triple arthrodeses) had a mean age of 58 years (18 to 84 ) at the time of surgery; 26 were in men and 74 in women. The diagnoses included posterior tibial tendon deficiency (PTTD) in 51, rheumatoid arthritis (RA) in 21, osteoarthritis (OA) in six and post-traumatic OA (PTOA) in 11. The diagnosis in the remaining 11 cases included psoriatic arthropathy, tarsal coalition, talipes, ochrinosis, poliomyelitis and Down's syndrome (Table I). All cases had been refractory to conservative therapy.

In 78 arthrodeses, fusion had been performed for painful flat feet (planovalgus), in 16 for arthritic symptoms (pain and stiffness) and in six for pain and deformity (three cavovarus, two equinovarus, one valgus; Table II).

The senior author (TSS) reviewed all the patients until fusion had occurred or a diagnosis of nonunion was established. CT was performed when nonunion was clinically suspected. 
Table I. Details of the pre-operative diagnoses in 100 triple arthrodeses

\begin{tabular}{lc}
\hline Diagnosis* & Number \\
\hline PTTD & 51 \\
RA & 21 \\
OA & 6 \\
PTOA & 11 \\
Coalition & 4 \\
PSA & 3 \\
Ochrinosis & 1 \\
Poliomyelitis & 1 \\
CTEV & 1 \\
Trisomy 21 & 1 \\
Total & 100 \\
\hline
\end{tabular}

* PTTD, posterior tibial tendon dysfunction; RA, rheumatoid arthritis; OA, osteoarthritis; PTOA, post-traumatic osteoarthritis; PSA, psoriatic arthropathy; CTEV, congenital talipes equinovarus
Table II. Details of the outcome according to diagnosis and deformity in 100 triple arthrodeses

\begin{tabular}{|c|c|c|c|c|c|}
\hline \multirow[b]{2}{*}{ Deformity } & \multirow[b]{2}{*}{ Diagnosis $^{*}$} & \multirow[b]{2}{*}{ Number } & \multicolumn{3}{|c|}{ Outcome } \\
\hline & & & Good & Fair & Poor \\
\hline \multirow[t]{7}{*}{ Planovalgus (78) } & PTTD & 51 & 40 & 10 & 1 \\
\hline & RA & 16 & 12 & $3^{\dagger}$ & 1 \\
\hline & OA & 3 & 1 & $1^{\dagger}$ & $1^{\ddagger}$ \\
\hline & PTOA & 3 & 2 & 1 & \\
\hline & Coalition & 2 & 1 & 1 & \\
\hline & PSA & 2 & 2 & & \\
\hline & Ochrinosis & 1 & 1 & & \\
\hline \multirow[t]{5}{*}{ Pain and stiffness (16) } & PTOA & 6 & 5 & 1 & \\
\hline & RA & 5 & 4 & 1 & \\
\hline & $\mathrm{OA}$ & 3 & 2 & & $1^{\dagger}$ \\
\hline & Coalition & 1 & & 1 & \\
\hline & PSA & 1 & 1 & & \\
\hline \multirow[t]{3}{*}{ Cavovarus (3) } & $O A$ & 1 & & & $1^{\S}$ \\
\hline & RA & 1 & 1 & & \\
\hline & PTOA & 1 & 1 & & \\
\hline \multirow[t]{2}{*}{ Equinovarus (2) } & Polio & 1 & 1 & & \\
\hline & CTEV & 1 & & 1 & \\
\hline Valgus (1) & Trisomy 21 & 1 & 1 & & \\
\hline Total & & 100 & 75 & 20 & 5 \\
\hline
\end{tabular}

* PTTD, posterior tibial tendon dysfunction; RA, rheumatoid arthritis; OA, osteoarthritis; PTOA, post-traumatic osteoarthritis; PSA, psoriatic arthroplasty; CTEV, congenital talipes equinovarus

$\dagger$ nonunion

‡ revised nonunion

$\S$ malunion

We contacted by telephone all the 33 patients who had been treated during the last four years of the study. They were asked whether they were satisfied with the outcome of the surgery, whether their symptoms had improved or deteriorated over time, and whether they would undergo the procedure again.

Operative technique. Two incisions were used. The subtalar and calcaneocuboid joints were exposed through a lateral incision, taking care to protect the peroneal tendons, sural nerve and superficial peroneal nerve. The talonavicular joint was approached through a medial incision. The joint surfaces were prepared using a flexible chisel which has only one side bevelled.

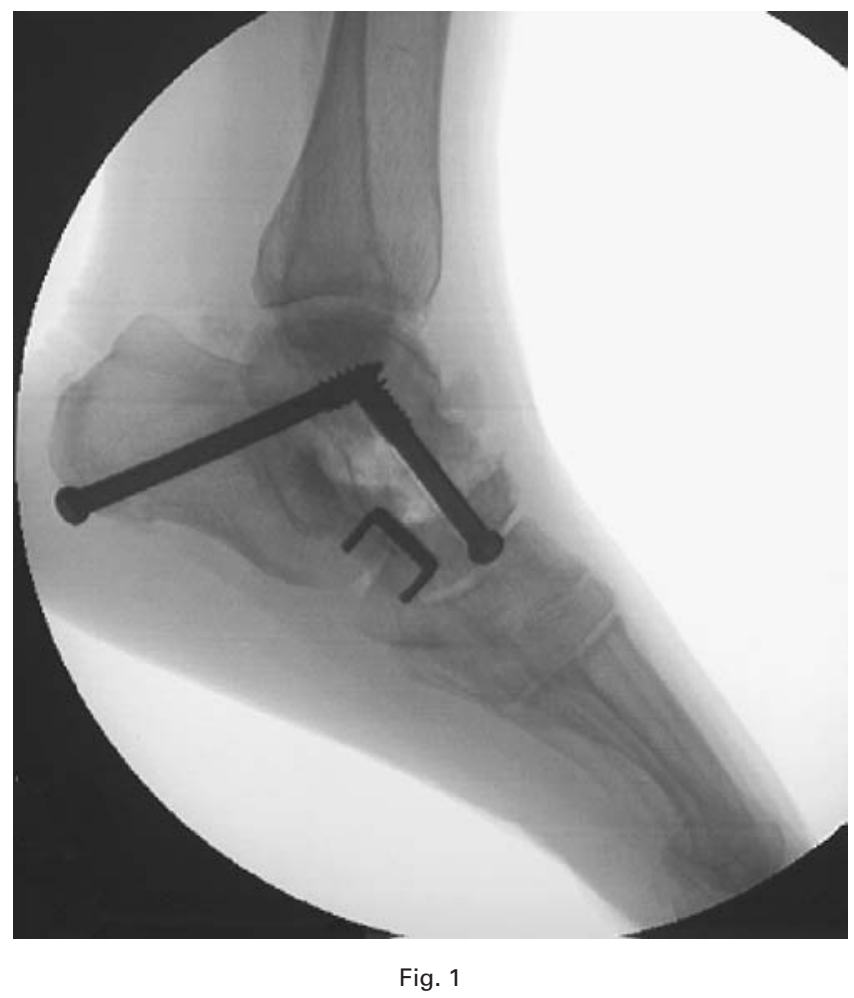

An intra-operative lateral image of a triple arthrodesis showing screw fixation of the subtalar and talonavicular joints with staple fixation of the calcaneocuboid joint.

The positioning of the foot is critical. Therefore, if neutral dorsiflexion could not be obtained, a percutaneous lengthening of tendo Achillis was performed (11). With the foot and hindfoot positioned, the talonavicular joint was fixed first using a 7.0-mm cannulated screw. The calcaneocuboid joint was stabilised by a staple. The subtalar joint was fixed by a single $7.0-\mathrm{mm}$ cannulated screw through the calcaneum into the talar dome under image-intensifier guidance (Fig. 1). Bone from the excised joint surfaces was the only material used for grafting. The subcutaneous tissues and skin were closed in layers and a below-knee backslab applied with the foot at a right angle.

After operation, the patients were reviewed at 10 to 14 days for inspection of the wound and the removal of sutures. At that stage, a full cast was applied for four weeks with no weight-bearing followed by a fully weight-bearing cast for a further six weeks.

Radiological assessment was made at six-weekly intervals until union had occurred. This was defined as pain-free weight-bearing with radiological evidence of union.

\section{Results}

All 96 patients were reviewed clinically and radiologically until union had occurred or nonunion diagnosed. The mean time to union was 5.1 months ( 3 to 17). The outcome was graded using the method of Angus and Cowell (Table III). ${ }^{2}$ 
Table III. The rating system of Angus and Cowell ${ }^{2}$

\begin{tabular}{ll}
\hline Rating & Signs and symptoms \\
\hline Good & No pain or minimal pain after heavy use \\
& No deformity or minimal deformity \\
& No callosities \\
& No pseudarthrosis \\
& No joint degeneration \\
& Pain after light use \\
Fair & Moderate deformity \\
& Single callosity \\
& Single pseudarthrosis \\
& Mild joint degeneration \\
& Pain on standing or at rest \\
Poor & Severe deformity \\
& Multiple callosities \\
& Multiple pseudarthrosis \\
& Severe joint degeneration
\end{tabular}

There were 75 good, 20 fair and five poor results. Of the five patients with poor results, in two the cause was nonunion. One of these required revision. In addition, there was one case of malunion which required revision, one of chronic infection and one of pain which required the wearing of custom shoes. A total of four patients in the series developed nonunion, two of whom had previously had an ankle fusion. The first was an 81-year-old woman with a planovalgus deformity and osteoarthritis below an ankle fusion performed 16 years previously. She continued to have limiting pain after a year, with nonunion of the subtalar joint. This was successfully revised using a fibular graft. The second was a 63-year-old woman with a previous ankle fusion. She developed nonunion of the subtalar joint at eight months, and has not required further surgery. The third was a 72-year-old woman with osteoarthritis and a planovalgus deformity. The calcaneocuboid joint failed to unite at 13 months. Her symptoms were mild and did not warrant further surgery. The fourth was a 58 -year-old woman with rheumatoid arthritis and a planovalgus deformity. She developed nonunion of the subtalar joint which was diagnosed at 12 months. Her symptoms were minor and she did not need further surgery.

Nine patients developed a wound infection. Eight were superficial and successfully treated with oral antibiotics. The one deep infection failed to respond to antibiotics, healing only after removal of all metalwork and debridement five months after operation. Four patients had delayed wound healing, one patient developed a deepvenous thrombosis and seven required removal of prominent screws. One patient had a symptomatic malunion which was successfully revised at two years.

We reviewed all 33 patients who had been treated in the last four years, two to five years after operation. One patient had died. Of the remaining 32 patients, 31 (97\%) were satisfied with the outcome of surgery. All would be prepared to undergo the procedure again, including the one dissatisfied patient. None had had any deterioration with time. The one dissatisfied patient required custom shoes and had a poor outcome. Our results are similar to those of previous studies. ${ }^{4}$

\section{Discussion}

Historically, triple arthrodesis has a high incidence of nonunion, occurring in up to $23 \%$ of cases. ${ }^{2}$ Wilson et al, ${ }^{1}$ when reviewing more than 300 triple arthrodeses performed between 1934 and 1959, reported a rate of nonunion of $10.3 \%$ with most occurring at the talonavicular joint.

The rates of nonunion have decreased significantly in recent studies, although bone grafting from the anterior iliac crest is common. Certainly some studies have recommended bone grafting in all cases. Cracchiolo et $\mathrm{al}^{7}$ achieved a rate of union of $100 \%$ with the use of an iliac crest dowel graft. Bednarz et $\mathrm{al}^{5}$ used iliac-crest graft in $89 \%$ of 63 triple fusions, obtaining a rate of nonunion of $4 \%$. Pell et $\mathrm{al}^{4}$ used supplementary bone graft in 53 of 132 arthrodeses. Initially, they used graft from the iliac crest or greater trochanter. However, they changed to taking graft from the talus and calcaneum because of problems at the donor site. Overall, they achieved a rate of nonunion of $2 \%$, although $31 \%$ of patients were lost to follow-up and not included in the study. Fortin and Walling ${ }^{6}$ achieved a rate of nonunion of $3 \%$ in 32 patients of whom 25 had grafts from the iliac crest and seven had allograft.

Some authors advise against using iliac-crest graft. Coughlin and Mann ${ }^{12}$ stated that bone grafting from the iliac crest was rarely necessary. Graves et $\mathrm{al}^{3}$ in their study on 17 triple fusions, performed using only local bone graft from the excised joint surfaces, had a rate of nonunion of $18 \%$. Bennet, Graham and Mauldin, ${ }^{13}$ presented the results of 25 triple arthrodeses performed using only local graft. The incidence of nonunion was $9 \%$ in 22 patients, although $12 \%$ were lost to follow-up.

Bone graft from the anterior iliac crest has a significant morbidity and complication rate, with chronic symptoms occurring in up to $26 \% .{ }^{10}$ Arrington et al ${ }^{11}$ reported the results of 414 iliac-crest bone-graft procedures. The overall rate of minor complications was $10 \%$, including superficial infections, seromas and minor haematomas. They defined a minor complication as that which responded to aggressive non-operative treatment. The rate of major complications was $5.8 \%$. This was defined as a complication which required a major change in treatment, prolonged hospitalisation or further surgery. Major complications included hernia at the donor site, vascular injury, nerve injury, deep infection, deep haematoma and fracture of the iliac wing. The vascular injuries all occurred with posterior iliac grafts.

Silber et $\mathrm{al}^{10}$ reviewed 134 patients two to six years after anterior iliac-crest bone grafting for cervical fusion. The patients were assessed by questionnaire or telephone interview. Within three months of surgery, $51 \%$ of patients had difficulty in walking and $8 \%$ to $12 \%$ reported chronic problems with daily activities. They highlighted the flaws in 
their study, using a retrospective questionnaire, but there were sufficient numbers for their findings to be valid.

There are several other sites available for bone grafts in the lower limb. These include the proximal and distant tibia, distal fibula, calcaneum, greater trochanter and distal femur. Complications occurring after bone has been taken from these sites occur less frequently, but include fracture through the donor site, the formation of a haematoma, infection, acute and chronic pain, and nerve injury. ${ }^{14}$

Our review of 100 consecutive cases with $100 \%$ followup gives a nonunion rate of $4 \%$. This has been achieved using a standard procedure with internal fixation using compression screws and staples. Local bone graft, from the excised joint surfaces, was used to fill any voids in the fusion surfaces. No other supplementary bone graft was used, thereby avoiding complications at the donor site. We note that the two patients who had a triple fusion beneath a previous ankle fusion, developed nonunion of the subtalar joint. This represents a rate of nonunion of $100 \%$. Under these circumstances, it is may be wise to augment the fusion with supplementary bone graft and additional fixation.

No benefits in any form have been received or will be received from a commercial party related directly or indirectly to the subject of this article.

\section{References}

1. Wilson F, Fay G, Lamotte P, Williams J. Triple arthrodesis: a study of factors affecting fusion after three hundred and one procedures. J Bone Joint Surg [Am] 1965; $47-A: 340-8$

2. Angus $\mathbf{P}$, Cowell H. Triple arthrodesis: a critical long term review. J Bone Joint Surg [Br] 1986;68-B:260-5.

3. Graves S, Mann R, Graves K. Triple arthrodesis in older adults: results after longterm follow-up. J Bone Joint Surg [Am] 1993;75-A:355-62.

4. Pell R 4th, Myerson MS, Schon IC. Clinical outcome after primary triple arthrodesis. J Bone Joint Surg [Am] 2000;82-A:47-57.

5. Bednarz P, Monroe M, Manoli A 2nd. Triple arthrodesis in adults using rigid internal fixation: an assessment of outcome. Foot Ankle Int 1999;20:356-63.

6. Fortin PT, Walling AK. Triple arthrodesis. Clin Orthop 1999;365:91-9.

7. Cracchiolo A 3rd, Pearson S, Kitaoka H, Grace D. Hindfoot arthrodesis in adults utilizing a dowel graft technique. Clin Orthop 1990;257:193-203.

8. Sangeorzan B, Smith D, Veith R, Hansen ST Jr. Triple arthrodesis using internal fixation in treatment of adult foot disorders. Clin Orthop 1993;294:299-307.

9. Alt V, Meeder P, Seligson D, Schad A, Atienza C Jr. The proximal tibia metaphysis: a reliable donor site for bone grafting? Clin Orthop 2003;414:315-21.

10. Silber JS, Anderson DG, Daffner SD, et al. Donor site morbidity after anterior iliac crest bone harvest for single-level anterior cervical discectomy and fusion. Spine 2003;28:134-9.

11. Arrington ED, Smith WJ, Chambers HG, Bucknell AL, Davino NA. Complications of iliac crest bone graft harvesting. Clin Orthop 1996;329:300-9.

12. Coughlin MJ, Mann RA. Surgery of the foot and ankle. Vol. 1. Seventh ed. St Louis: Mosby, 1999:682-8.

13. Bennet GL, Graham CE, Mauldin DM. Triple arthrodesis in adults. Foot Ankle 1911;12:138-43.

14. Boone D. Complications of iliac crest graft and bone grafting alternatives in foot and ankle surgery. Foot Ankle Clin 2003:8:1-14. 